\title{
A NOTE ON THE BANACH-MAZUR PROBLEM
}

\author{
BEATA RANDRIANANTOANINA ${ }^{\dagger}$ \\ Department of Mathematics and Statistics, Miami University, Oxford, OH 45056, USA \\ e-mail: randrib@muohio.edu
}

(Received 12 June, 2000; revised 15 September, 2000)

\begin{abstract}
We prove that if $X$ is a real Banach space, with $\operatorname{dim} X \geq 3$, which contains subspace of codimension 1 which is 1-complemented in $X$ and whose group of isometries is almost transitive then $X$ is isometric to a Hilbert space. This partially answers the Banach-Mazur rotation problem and generalizes some recent related results.
\end{abstract}

2000 Mathematics Subject Classification. 46C15, 46B04, 46B20.

1. Introduction. In 1930's Banach and Mazur [2] (see also [20, Problem 9.6.2]) posed a problem whether every separable Banach space with transitive group of isometries has to be isometric to a Hilbert space. Here we say that the group of isometries of a Banach space $X$ is transitive if for every $x, y \in X$ with $\|x\|=\|y\|=1$ there exists an isometry $T: X \stackrel{\text { onto }}{\longrightarrow} X$ such that

$$
T x=y \text {. }
$$

Mazur [16] answered this problem positively for finite dimensional Banach spaces $X$ and Pełczyński and Rolewicz [18] showed that the answer is negative when $X$ is not assumed to be separable. The case of infinite dimensional separable spaces remains open despite active research in the area, see [20] and a recent survey [9].

Closely related to the notion of transitivity are the notions of almost transitivity and convex transitivity. We say that a group of isometries of $X$ is almost transitive (resp. convex transitive) if for every $x$ in the unit sphere of $X, S_{X}=\{x:\|x\|=1\}$ the orbit of $x$ i.e. the set $G_{x}=\{T x: T$ isometry of $X\}$ is dense in $S_{X}\left(\operatorname{resp} \cdot \operatorname{conv}\left(G_{x}\right)\right.$ is dense in $S_{X}$ ). Sometimes we will abuse language and say that a space $X$ is almost transitive (resp. convex transitive or transitive) provided the group of isometries of $X$ is almost transitive (resp. convex transitive or transitive).

Spaces with almost transitive and convex transitive groups of isometries have been actively studied. It is known that there exist non-Hilbertian separable Banach spaces with almost transitive groups of isometries, for example $L_{p}[0,1], 1 \leq p<\infty$, are such spaces [18], see also [20] and see [10] for detailed study which function spaces are almost transitive. Several questions have been posed to find additional conditions on a Banach space $X$ which together with almost transitivity, or with just convex transitivity, imply that $X$ is isometric to a Hilbert space. Maybe the most famous conjecture of this type is the conjecture of Wood [23] that if $C_{0}(L)$ is almost transitive in its natural supremum norm then $L$ is a singleton, i.e. $C_{0}(L)$ is one dimensional. Wood's conjecture is still open despite recent active research in the area, see [9].

Participant, NSF Workshop in Linear Analysis and Probability, Texas A\&M University, College Station, Texas. 
The main theorem of the present paper is the following:

Theorem 1.1. (see Theorem 2.7 below). Suppose that $X$ is a real Banach space, with $\operatorname{dim} X \geq 3$, which contains a 1-complemented hyperplane and whose group of isometries is almost transitive. Then $X$ is isometric to a Hilbert space.

Our method of proof relies on the theory of numerical ranges [6,7]. We postpone the proof to the next section and now we will discuss the connections with existing results in the literature.

Note that spaces $L_{p}[0,1], 1 \leq p<\infty$, do not have 1-complemented hyperplanes (see e.g. $[\mathbf{1 2}, \mathbf{1 9}])$ and, as mentioned above, they are almost transitive.

Theorem 1.1 generalizes a recent result of Skorik and Zaidenberg:

Theorem 1.2. [22] Suppose that $X$ is a real Banach space which contains an isometric reflection and whose group of isometries is almost transitive. Then $X$ is isometric to a Hilbert space.

Here we say that an operator $T$ is a reflection on $X$ if there exist $e \in X, e^{*} \in X^{*}$ with $e^{*}(e)=1$ so that

$$
T(x)=x-2 e *(x) e .
$$

If this happens we write $T=S_{e, e^{*}}$, and if $S_{e, e^{*}}$ is an isometry we say that $e$ is an isometric reflection vector in $X$.

To see that Theorem 1.1 is more general than Theorem 1.2 we observe that if a space $X$ admits an isometric reflection operator then $X$ contains a 1-complemented hyperplane but not vice-versa.

Indeed, assume that for some $e \in S_{X}$ there exists $e^{*} \in X^{*}$ with $e^{*}(e)=1$ so that an operator $S_{e, e^{*}}: x \mapsto x-2 e^{*}(x) e$ is an isometric reflection in $X$. Then for all $x \in X$ we have:

$$
\left\|x-e^{*}(x) e\right\|=\left\|\frac{1}{2}\left(\left(x-2 e^{*}(x) e\right)+x\right)\right\| \leq \frac{1}{2}\left(\left\|x-2 e^{*}(x) e\right\|+\|x\|\right)=\|x\|
$$

Thus $x \mapsto x-e^{*}(x) e$ is a contractive projection in $X$ onto the hyperplane $\operatorname{Ker}\left(e^{*}\right)$ $\subset X$.

On the other hand consider the two dimensional real space $X$ whose unit sphere is the convex hull of the points $(1,0),(1 / 2,1),(-1,1),(-1,0),(-1 / 2,-1),(-1,-1)$ as sketched below.

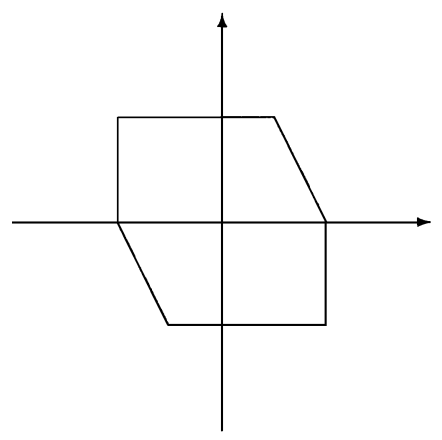


Then $X$ contains no isometric reflections but clearly every hyperplane is 1-complemented in $X$.

It is not difficult to construct spaces of arbitrary dimension which contain no isometric reflections but which do contain 1-complemented subspaces of codimension 1.

Statements similar to Theorem 1.2 have been recently studied by J. Becerra Guerrero, F. Cabello Sanchez and A. Rodriguez Palacios. F. Cabello Sanchez [8] showed that Theorem 1.2 is valid in complex Banach spaces. J. Becerra Guerrero and A. Rodriguez Palacios linked this result with the following characterization of Hilbert spaces due to Berkson [5] and Kalton and Wood [13]:

Theorem 1.3. Let $X$ be a complex Banach space. If $x \in X$ is such that $\operatorname{span}\{x\}$ is the range of a hermitian projection in $X$ then $x$ is called a hermitian element in $X$.

If every nonzero element of $X$ is hermitian in $X$ then $X$ is a Hilbert space.

J. Becerra Guerrero and A. Rodriguez Palacios [3] observed that an element $x \in X$ is hermitian in $X$ if and only if $x$ is an isometric reflection vector in $X$. Thus in the complex case we have the following stronger version of Theorem 1.2:

Theorem 1.4. [13, Theorem 6.4] Let $X$ be a complex Banach space. If $X$ is convex transitive and $X$ contains an isometric reflection vector then $X$ is a Hilbert space.

J. Becerra Guerrero and A. Rodriguez Palacios generalized Theorem 1.2 as follows:

TheORem 1.5. [4] Let $X$ be a real or complex Banach space. If there exists a nonrare set in $S_{X}$ consisting of isometric reflection vectors then $X$ is a Hilbert space.

We do not know whether Theorem 1.1 can be generalized for convex transitive spaces (to obtain an analogue of Theorem 1.4). However the following example illustrates that an analogue of Theorem 1.5 for norm-one complemented hyperplanes fails in a very strong way. Namely we have:

ExAmple 1.6. For every $\varepsilon>0$ there exists a 3-dimensional Banach space $X_{\varepsilon}$ which is not isometric to a Hilbert space and such that the set $F$ of functionals $f \in S_{X_{\varepsilon}^{*}}$ with Ker $f$ norm-one complemented in $X_{\varepsilon}$ is open in $S_{X_{\varepsilon}^{*}}$ and $\mu\left(S_{X_{\varepsilon}^{*}} \backslash F\right)<\varepsilon$, (here $\mu$ denotes the Lebesgue measure on $S_{X_{\varepsilon}^{*}} \subset \mathbb{K}^{3}, \mathbb{K}=\mathbb{R}$ or $\mathbb{C}$ ).

Moreover $X_{\varepsilon}$ can be chosen to be uniformly convex.

Proof. Let $\varepsilon>0$. Fix $\delta>0$ so that

$$
\mu\left(\left\{\left(x_{1}, x_{2}, x_{3}\right) \in S_{\ell_{2}}:\left|x_{3}\right| \geq 1-\delta\right\}\right)<\varepsilon .
$$

Let $\varphi:\left(\mathbb{R}_{+}\right)^{3} \rightarrow \mathbb{R}$ be a convex continuous function such that

$$
\varphi\left(t_{1}, t_{2}, t_{3}\right) \begin{cases}=1 & \text { if }\left(t_{1}, t_{2}, t_{3}\right)=(0,01) \\ =\sqrt{t_{1}^{2}+t_{2}^{2}+t_{3}^{2}} & \text { if } t_{3} \leq(1-\delta) \sqrt{t_{1}^{2}+t_{2}^{2}+t_{3}^{2}} \\ >\sqrt{t_{1}^{2}+t_{2}^{2}+t_{3}^{2}} & \text { if } t_{3}>(1-\delta) \sqrt{t_{1}^{2}+t_{2}^{2}+t_{3}^{2}}\end{cases}
$$


We can additionally require $\varphi$ to have any desired degree of smoothness.

We define a norm on $\mathbb{R}^{3}$ using function $\varphi$ :

$$
\left\|\left(x_{1}, x_{2}, x_{3}\right)\right\|_{X_{\varepsilon}}=\varphi\left(\left|x_{1}\right|,\left|x_{2}\right|,\left|x_{3}\right|\right)
$$

Then $S_{X_{\varepsilon}} \subseteq S_{\ell_{2}}$ and $S_{X_{\varepsilon}^{*}} \cap S_{l_{2}} \supset\left\{\left(x_{1}, x_{2}, x_{3}\right) \in S_{l_{2}}:\left|x_{3}\right| \leq 1-\delta\right\}$ as illustrated in the figure below.

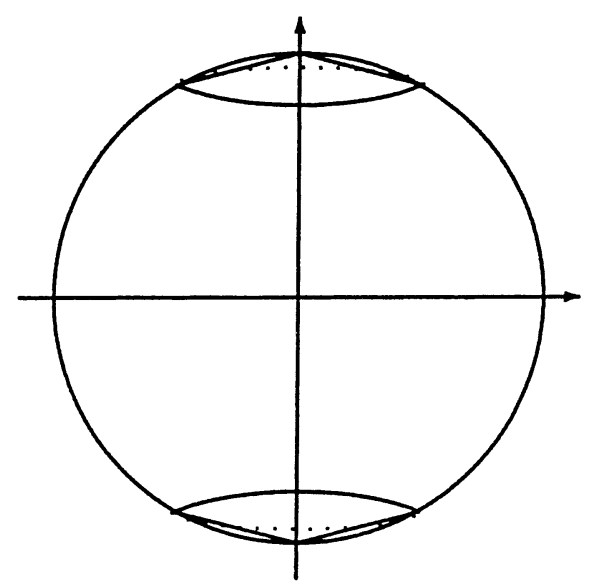

Thus, if $f \in S_{X_{\varepsilon}^{*}} \cap S_{\ell_{2}}$ then Ker $f \cap S_{X_{\varepsilon}} \subset S_{\ell_{2}}$ and the orthogonal projection $P$ onto Ker $f$ has norm 1 in $X_{\varepsilon}$. Hence

$$
F=\left\{f \in S_{X_{\varepsilon}^{*}}: \operatorname{Ker} f \text { is 1-complemented in } X_{\varepsilon}\right\} \supset S_{X_{\varepsilon}^{*}} \cap S_{\ell_{2}} .
$$

Thus $\mu\left(S_{X_{\varepsilon}^{*}} \backslash F\right)<\varepsilon$, as desired.

Theorem 1.1 relies on the real analogue of the notion of hermitian elements (see Definitions 1 and 2 below) whose existence in $X$ is equivalent to the existence of norm one complemented hyperplanes in $X$. We note here that our proof is much shorter than the existing proofs of Theorem 1.2.

2. Proofs of main results. We begin with definitions of real analogues of hermitian elements which were introduced by Kalton and the author [12] based on ideas of P. H. Flinn, cf. [21].

Definition 1. [21] We say that an operator $T: X \rightarrow X$ is numerically positive if for all $x \in X$ there exists a $x^{*} \in X^{*}$ or, equivalently, for all $x^{*} \in X^{*}$ with $\left\|x^{*}\right\|^{2}=\|x\|^{2}=x^{*}(x)$ and $x^{*}(T x) \geq 0$, i.e. the numerical range of $T$ is contained in $\mathbb{R}_{+} \cup\{0\}$ (cf. $\left.[6,14]\right)$.

Definition 2. [12] (based on ideas of P. H. Flinn [21]) We say that $u \in X$ is $a$ Flinn element if there exists a numerically positive projection $P: X \stackrel{\text { onto }}{\longrightarrow} \operatorname{span}\{u\}$ i.e. if there exists $f \in X^{*}$ with $f(u)=1$ and such that the map $f \otimes u$, defined by $x \mapsto f(x) u$, is numerically positive. We say then that $(u, f)$ is a Flinn pair. 
The set of all Flinn elements of $X$ will be denoted by $\mathcal{F}(X)$.

We list few straightforward properties of Flinn elements which are important for the future use. We include their short proofs for completeness.

Proposition 2.1. [21, Lemma 1.4] A projection $P: X \rightarrow X, P \neq I$, is numerically positive if and only if $\|I-P\|=1$.

Proof. If $\|I-P\|=1$ and $x \in X, x^{*} \in X^{*}$ are such that $1=\left\|x^{*}\right\|^{2}=\|x\|^{2}=x^{*}(x)$, then

$$
1 \geq x^{*}((I-P)(x))=1-x^{*}(P(x)) .
$$

Thus $x^{*}(P(x)) \geqslant 0$ and $P$ is numerically positive.

To see the implication in the other direction we rely on the result of Lumer and Phillips [15] that operator $P$ is numerically positive if and only if $\|\exp (-t P)\| \leq 1$ for all real $t \geq 0$. We have for all real $t \geq 0$ :

$$
\exp (-t P)=I+\sum_{j=1}^{\infty} \frac{(-t)^{j} P^{j}}{j !}=I+\left(\sum_{j=1}^{\infty} \frac{(-t)^{j}}{j !}\right)=I+\left(e^{-t}-1\right) P
$$

where the second equality holds because $P$ is a projection. Thus by result of Lumer and Phillips if $P$ is numerically positive we have

$$
\|I-P\|=\lim _{t \rightarrow \infty}\|\exp (-t P)\| \leq 1
$$

Since $P \neq I$ and $I-P$ is a projection we get $\|I-P\|=1$.

Proposition 2.2. [12, Proposition 3.2] Suppose that $T: X \rightarrow X$ is a surjective isometry. Then $T(\mathcal{F}(X))=\mathcal{F}(X)$.

Proof. If $u \in \mathcal{F}(X) \backslash\{0\}$ then there exists $f \in X^{*}$ such that the projection $P: X \rightarrow \operatorname{span}\{u\}$ defined by $x \mapsto f(x) u$ is numerically positive. Then the projection $Q: x \mapsto\left(\left(T^{*}\right)^{-1} f\right)(x) . T(u)$ establishes the fact that $T(u)$ is Flinn.

Proposition 2.3. [12, Proposition 3.1] The set $\mathcal{F}(X)$ is closed.

Proof. Suppose $u_{n} \in \mathcal{F}(X)$ with $\lim \left\|u_{n}-u\right\|=0$. It suffices to consider the case when $\left\|u_{n}\right\| \neq 0$ and $\|u\| \neq 0$. Then there exist $f_{n} \in X^{*}$ so that $f_{n} \otimes u_{n}$, is a numerically positive projection. Thus $\left\|f_{n} \otimes u_{n}\right\|=\left\|f_{n}\right\|\left\|u_{n}\right\| \leq 2$. Thus $\left\|f_{n}\right\| \leq 2 \sup \left(1 /\left\|u_{n}\right\|\right)$. By Alaoglu's theorem $\left(f_{n}\right)$ has a weak*-cluster point $f$ and clearly $(u, f)$ is a Flinn pair.

Proposition 2.4. Suppose $u \in \mathcal{F}(X)$ and that $Y$ is a subspace of $X$ such that $u \in Y$. Then $u \in \mathcal{F}(Y)$.

Proof. Without loss of generality $u \neq 0$. Since $u \in F(X)$ there exists $f \in X^{*}$ with $f(u)=1$ and such that the map $x \mapsto f(x) u$ is numerically positive. Consider $g=\left.f\right|_{Y} \in Y^{*}$ and the map $Q: Y \rightarrow \operatorname{span}\{u\} \subset Y$ defined by

$$
Q(y)=g(y) u
$$


By Hahn-Banach Theorem for every $y \in Y$ and every $y^{*} \in Y^{*}$ with $\left\|y^{*}\right\|^{2}=\|y\|^{2}=$ $y^{*}(y)$ there exists $\tilde{y}^{*} \in X^{*}$ with $\left\|\tilde{y}^{*}\right\|=\left\|y^{*}\right\|=\|y\|$ and $\left.\tilde{y}^{*}\right|_{Y}=y^{*}$. Thus we get, since $(u, f)$ is a Flinn pair in $X$ :

$$
g(y) y^{*}(u)=f(y) \tilde{y}^{*}(u) \geq 0
$$

and $g(u)=f(u)=1$. Hence $(u, g)$ is a Flinn pair in $Y$.

Now we are ready for the real analogue of Theorem 1.3 ([5, Theorem 2.22], [13, Corollary 4.4]).

Theorem 2.5. Suppose that $X$ is a real Banach space $\operatorname{dim} X \geq 3$ and $(X)=X$. Then $X$ is isometric to a Hilbert space.

Proof. Since Hilbert spaces are characterized by the parallelogram identity it is enough to show that the result holds for all 3-dimensional subspaces of $X$ (cf. $\left[1,\left(1.4^{\prime}\right)\right]$ ). Suppose that $Y \subset X$ is a real Banach space with $\operatorname{dim} Y=3$. Then, by Proposition 2.4, $\mathcal{F}(Y)=Y$ i.e. for every $u \in Y$ there exists an $f \in Y^{*}$ such that $f(u)=1$ and the map $f \otimes u$ is a numerically positive projection in $Y$, and also in $Y^{*}$.

Hence, by Proposition 2.1, the map $I-f \otimes u$ is a contractive projection of $Y^{*}$ onto $\operatorname{Ker} u \subset Y^{*}$. Since $\operatorname{dim} Y=3$, we conclude that every 2-dimensional subspace of $Y^{*}$ is contractively complemented. Thus $Y^{*}$ is isometric to a Hilbert space by the following criterion due to Kakutani:

Theorem 2.6. [11] (see also [1, p. 99]) Suppose that $Z$ is a Banach space of dimension at least 3. Then $Z$ is isometric to a Hilbert space if and only if for every 2-dimensional subspace $F$ of $Z$ there is a norm one linear projection $P: Z \rightarrow F$.

Therefore $Y$ is isometric to Hilbert space and the proof is finished.

Next is our main theorem.

Theorem 2.7. Suppose that $X$ is a real Banach space, with $\operatorname{dim} X \geq 3$, which contains a 1-complemented hyperplane and whose group of isometries is almost transitive. Then $X$ is isometric to a Hilbert space.

Proof. When $X$ contains a subspace of codimension 1 which is 1-complemented in $X$ then, by Proposition 2.1, $X$ contains a nonzero Flinn element. Let $u \in S_{X}$ be a Flinn element. By Proposition 2.2

$$
G_{u}=\{T u: T \text { isometry of } X \text { onto } X\} \subset \mathcal{F}(X)
$$

and by almost transitivity of $X, G_{u}$ is dense in $S_{X}$. Since, by Proposition $2.3, \mathcal{F}(X)$ is closed we obtain:

$$
F(X) \cap S_{X}=S_{X}
$$

Thus, $\mathcal{F}(X)=X$ and the result follows by Theorem 2.5 . 
Acknowledgements. I wish to thank Professor G. Wood for telling me about the current status of work on problems related to Banach-Mazur problem, and to Professors F. Cabello Sanchez and A. Rodriguez Palacios for providing me with copies of their preprints.

Added in proof. After this paper has been completed and accepted for publication P.L. Papini has pointed out to me the reference [17] which contains a result analogous to Theorem 1.1 with the additional assumptions that $X$ is reflexive and transitive.

\section{REFERENCES}

1. D. Amir, Characterizations of inner product spaces (Birkhäuser-Verlag, 1986).

2. S. Banach, Theorie des operations lineaires, Monografie Mat. 1 (Polish Scientific Publishers, Warsaw, 1932).

3. J. Becerra Guerrero and A. Rodriguez Palacios, Isometric reflexions on Banach spaces after a paper of Skorik and Zaidenberg, Rocky Mountain J. Math., to appear.

4. J. Becerra Guerrero and A. Rodriguez Palacios, Isometries which are one-dimensional perturbations of the identity, Quart. J. Math. Oxford Ser (2) 50 (1999), 147-153.

5. E. Berkson, Hermitian projections and orthogonality in Banach spaces, Proc. London Math. Soc. (3) 24 (1972), 101-118.

6. F. F. Bonsall and J. Duncan, Numerical ranges of operators on normed spaces and of elements of norrmed algebras London Mathematical Society Lecture Note Series, No. 2 (Cambridge University Press, 1971).

7. F. F. Bonsall and J. Duncan, Numerical ranges II London Mathematical Society Lecture Note Series, No. 10 (Cambridge University Press, 1973).

8. F. Cabello Sánchez, 10 Variaciones sobre un terna de Mazur, Tesis Doctoral (Universidad de Extremadura, 1996).

9. F. Cabello Sánchez, Regards sur le problème des rotations de Mazur, Extracta Math. 12 (1997), 97-116.

10. P. Greim, J. E. Jamison, and A. Kamińska, Almost transitivity of some function spaces, Math. Proc. Cambridge Philos. Soc. 116 (1994), 475-488. [Corrigendum: Math. Proc. Cambrigde Philos. Soc. 121 (1997), 191-192]. 93-97.

11. S. Kakutani, Some characterizations of Euclidean space, Japan J. Math. 16 (1939),

12. N. J. Kalton and B. Randrianantoanina. Surjective isometries of rearrangementinvariant spaces, Quart. J. Math Oxford Ser (2) 45 (1994), 301-327.

13. N. J. Kalton and G. V. Wood, Orthonormal systems in Banach spaces and their applications, Math. Proc. Camb. Phil. Soc. 79 (1976), 493-510.

14. G. Lumer, Semi-inner product spaces, Trans. Amer. Math. Soc. 100 (1961), 29-43.

15. G. Lumer and R. S. Phillips, Dissipative operators a Banach space, Pacific J. Math. 11 (1961), 679-698.

16. S. Mazur, Quelques propriétés des espaces euclideans, C. R. Acad. Sci Paris 207 (1938), 761-764.

17. V. P. Odinec, On a property of reflexive Banach spaces with a transitive norm, Bull Acad. Polon. Sci. Sér. Sci. Math. 30 (1982), 353-357.

18. A. Pełczyński and S. Rolewicz, Best norms with respect to isometry groups in normed linear spaces, Short Communications on International Math. Congress in Stockholm (1962), 104.

19. B. Randrianantoanina, Contractive projections in nonatomic function spaces, Proc. Amer Math Soc. 123 (1995), 1747-1750.

20. S. Rolewicz, Metric linear spaces (Polish Scientific Publishers, Warsaw, 1972).

21. H. P. Rosenthal, Contractively complemented subspaces of Banach spaces with reverse monotone (transfinite) bases. Longhorn Notes, The University of Texas Functional Analysis Seminar, (1984-5), 1-14.

22. A. Skorik and M. G. Zaidenberg. On isometric reflections in Banach spaces, Mat. Fiz. Anal. Geom. 4 (1997), 212-247.

23. G. V. Wood, Maximal symmetry in Banach spaces, Proc. Roy. Irish Acad. Sect. A 82 (1982) ,177-186 